\title{
FURIHATA Setsuo \\ Entwicklung des japanischen Kapitalismus und marxistische Wirtschaftswissenschaft in Japan
}

\section{Die Entwickhung des japanischen Kapitalismus vor dem Zweiten Welthrieg}

Die Ankunft des Kommandeurs der Indischen Flotte der Vereinigten Staaten, Perry, mit vier Kriegsschiffen im Jahre 1853 versetzte der japanischen Feudalgesellschaft, die mehr als 220 Jahre abgeschlossen war, einen kräftigen Schlag: fortan war sie der westlichen Zivilisation ausgesetzt. 1858 schioß Japan mit fünf Staaten, darunter den USA, England und Frankreich, Handesverträge ab und öffnete die Häfen Yokohama, Nagasaki und Hakodate. Damit wurde Japan gewaltsam in die kapitalistische Welt hineingezogen und erlebte nun einen stürmischen Handels-Boom. Dieser Handels-Boom Iöste in der japanischen Wirtschaft eine kräftige Inflation aus und führte zur Verelendung der bäuerlichen und sonstigen arbeitenden Bevölkerung. Das Ergebnis war eine Revolution, deren Hauptkraft die mit dem Tokugawa-Regime unzufriedene untere Kriegerklasse bildete. Der Slogan der Revolutionäre lautete anfangs »Ehrt den Kaiser und vertreibt die Barbaren!«, aber als man 1863 im Krieg zwischen dem auf Kyushu gelegenen Lehnsfürstentum Satsuma und England die Kraft des europäischen Kapitalismus zu spüren bekommen hatte, trat an seine Stelle der Slogan »Öffnet das Land und stürzt das Tokugawa-Regime! «Folglich riß die 1867 errichtete Meiji-Regierung die feudalen Gesellschaftsinstitutionen nieder und verwandte alle Kraft darauf, die Voraussetzungen für die Entwicklung des Kapitalismus zu schaffen. Zuerst beseitigte man das feudale Ständesystem und die Beschränkungen für Orts- und Berufswechsel. Um die Privilegien der Krieger abzuschaffen, wurde die erbliche Besoldung von Haushalten der Kriegerklasse abgeschafft, eine Grundsteuer-Reform durchgeführt und das Verbot des Kaufs und Verkaufs von Reisanbauflächen aufgehoben; auch die Beschränkungen des kommerziellen Güterverkehrs entfielen. Wenn der Grundbesitz frei wird, das Ständesystem abgeschafft und die Freiheit der Berufswahl garantiert werden, so sind damit die sozialen Bedingungen für die Entwicklung kapitalistischer Verhältnisse gegeben.

Nach der Restauration des Kaisertums 1867 wurde von der neuen Regierung sofort das Bankwesen nach westlichem Muster übernommen, ein Eisenbahnnetz aufgebaut und eine landesweite Post etabliert. Von der Regierung wurden auch Rüstungsarsenale, Werften, Bergwerke etc. betrieben sowie Musterfabriken errichtet. Auf diese Weise bildeten sich zwischen dern Chinesisch-Japanischen (1894-95) und dem Russisch-Japanischen Krieg (190405) kapitalistische Verhältnisse in Japan heraus. 1897, als mit Hilfe der Reparationszahlungen aus dem Chinesisch-Japanischen Krieg der Goldstandard eingeführt werden konnte, ist als das Jahr der Etablierung des japanischen Kapitalismus anzusehen.

Die Reproduktionsstruktur des so entstandenen japanischen Kapitalismus läßt sich nun folgendermaßen zusammenfassen: Die erwerbstätige Bevölkerung arbeitete im Jahre 1900 zu $65 \%$ in der Land- und Forstwirtschaft und in der Fischerei; 35\% waren in nicht-agrari- 
schen Sektoren beschäftigt, davon lediglich 4\% in modernen Wirtschaftszweigen. Die bedeutendsten Branchen innerhalb der Industrie waren die Seidenindustrie (Rohseide) sowie die Spinnerei und Weberei (Baumwolle). Was die Baumwollweberei betrifft, so wurde diese in Japan derart entwickelt, daß man nach dem Ersten Weltkrieg in der Produktion von Baumwollstoffen das bis dahin in der Welt führende England überholt hatte. Demgegenüber wurden im schwerindustriellen Sektor fast nur Regierungsunternehmen betrieben wobei die Rüstungsindustrie im Mittelpunkt stand; die internationale Konkurrenzfähigkeit war hier äußerst schwach. Die Bauern, die mehr als die Hälfte der Bevölkerung ausmachten, produzierten also Reis und Seidenraupen (Kokons); die gewonnene Rohseide wurde fast vollständig nach Nordamerika exportiert. Mit dem Exporterlös wurde der Import von Rohbaumwolie bezahlt, und die Verarbeitung dieser Rohbaumwolle und der Export fertiger Baumwollerzeugnisse bildeten die wichtigste Aktivität der kapitalistischen Unternehmen. Die Beherrschung des asiatischen Marktes mittels dieser Baumwollprodukte war der Motor des japanischen Imperialismus. In den zwanziger und dreibiger Jahren dieses Jahrhunderts konnte man Japan mit seiner hier angedeuteten spezifischen Industriestruktur bereits zu den wichtigen imperialistischen Staaten der Welt zählen - zusammen mit England, den USA, Deutschland und Frankreich.

\section{Die Entwicklung des japanischen Marxismus}

In Japan begann die Entwicklung des Kapitalismus zu einem Zeitpunkt, als er in anderen Ländern bereits das Stadium des Imperialismus erreicht hatte. Daher war Japan zu einer rapiden Beschleunigung der kapitalistischen Entwicklung gezwungen, in der die Regierung die Initiative hatte, unter der Zielsetzung »macht das Land reich und die Armee stark! « So sollte sich das Land gegen die westlichen Mächte behaupten. Dementsprechend wurde zwar nach den Meiji-Reformen ${ }^{1}$ die klassische Ökonomie eines A. Smith, J. S. Mill und anderer in Japan bekanntgemacht, doch die Ideen des Liberalismus konnten in einer durch staatliche Protektionspolitik und die Herausbildung von Monopolkapitalen in Form von Aktiengesellschaften charakterisierten Situation nicht Fuß fassen. Vielmehr griff man bereitwillig die Thesen der deutschen historischen Schule auf, in denen der nationale Charakter Deutschlands, des »Nachzüglerlandes«, im Gegensatz zum englischen Liberalismus betont wurde: unter dem Einfluß der Neuen historischen Schule der Nationalökonomie Gustay Schmollers und Lujo Brentanos wurde der »Japanische Verein für Sozialpolitik « gegründet, der fortan die Hauptströmung in der Wirtschaftswissenschaft darstellte.

Im Zuge der rapiden Entwicklung des Kapitalismus wurden die »Arbeiterfrage« und die »Bauernfrage« natürlich zugespitzt; da außerdem der Chinesisch-Japanische und der Russisch-Japanische Krieg in einem Teil der Bevölkerung eine starke antimilitaristische Stimmung hatten entstehen lassen, kam es aufgrund dieser verschiedenen Faktoren dazu, daß sozialistische Ideen in Japan Anhänger fanden. Im Jahre 1901 wurde die Sozial-Demokratische Partei von Shusui Kotoku (1871-1911), Sen Katayama (1859-1933) u.a. gegründet; damit begann auch die schrittweise Rezeption der Theorie des Marxismus durch ein breiteres Publikum. Natürlich wurde die Sozial-Demokratische Partei noch am Tage ihrer Gründung ver- 
boten, aber im Jahre 1904 erschien in der »Heimin-Shinbun « eine japanische Übersetzung des »Kommunistischen Manifests«, und 1907 wurde in der »Osaka-Heimin-Shinbun « der Inhalt des »Kapital in einer Zusammenfassung von Hitoshi Yamakawa (1880-1958) vorgestellt. Auf der einen Seite konnte man somit eine rasche Verbreitung der Ideen und der Theorie des Marxismus feststellen, auf der anderen Seite setzte eine scharfe Repression gegen die sozialistische Bewegung ein: deren »Winterzeit« begann mit dem Todesurteil gegen Kotoku und zwölf andere im Jahre 1910; die Begründung für dieses Urteil lautete, diese härten einen »Plan zur Ermordung des Tenno« zu realisieren versucht.

Der Erste Weltkrieg brachte eine schnelle Expansion des japanischen Kapitalismus: zwischen 1914 und 1919 nahm das Kapital für Unternehmensneugründungen und -erweiterungen für die Industrie insgesamt um das 16 -fache zu; für die Metallindustrie läßt sich ein außergewöhnliches Anwachsen um das 34-fache und für den Schiffbau gar um das 112 -fache belegen. Aber Rückschläge blieben nicht aus: Japan geriet mehriach hintereinander in ökonomische Notlagen -- die Nachkriegskrise 1920, das große Erdbeben im Kanto-Gebiet um Tokyo 1923, die Finanzkrise 1927 und die Weltwirtschafskrise 1929 ließen überall in Stadt und Land Arbeiterkämpfe und Streiks der Pachtbauern aufflammen. Besonders die sozialistische Bewegung erholte sich unter dem Einfluß der Russischen Revolution wieder und breitete sich aus.

Diese sozialistische Bewegung war bereits nicht mehr wie früher ein lockerer Zusammenschluß christlicher, humanistischer und syndikalistischer Gruppierungen, sondern hatte die Form einer fest organisierten Bewegung angenommen, die sich maßgeblich auf den Marxismus berief. 1926 wurden die Soziale-Massen-Partei und die Japanische Arbeiterund Bauern-Partei gegründet; aber die Hauptströmung in der sozialistischen Bewegung bildete die Kommunistische Partei Japans (KPJ), die 1922 gegründet und, obwohl 1924 zeitweilig aufgelöst, 1926 reorganisiert wurde. Ab 1927 war sie als Untergliederung der Komintern aktiv. Die Komintern beschloß damals dreierlei »Thesen «, die als Analyse von Politik und Wirtschaft Japans und als Kompaß für die Bewegung dienen sollten: die $\gg$ Resolution zu Japan « (Thesen von 1927), den »Entwurf der politischen Thesen der Kommunistischen Partei Japans (Thesenentwurf von 1931), und die »Thesen zur Lage in Japan und den Aufgaben der Kommunistischen Partei Japans« (Thesen von 1932). Die Thesen von 1927 und 1932 hatten im großen und ganzen denselben Inhalt; demgegenüber wies der Thesenentwurf von 1931 beträchtliche Unterschiede auf. Algemein gesprochen, bestimmten erstere Japan als absolute Monarchie und erklärten eine bürgerliche Revolution zum anstehenden politischen Ziel, während in letzterer Japan als »bürgerlicher Staat« bezeichnet und als politisches Ziel die proletarische Revolution festgelegt wurde. In der Komintern wurde jedoch, parallel zur Etablierung des Stalinschen Herrschaftssystems, dieser Thesenentwurf von 1931 als »japanische Ausgabe des Trotzkismus« abgestempelt und zurückgezogen, so daß die Thesen von 1932 als die orthodox-marxistische Position zu Japan schließlich maßgeblich wurde.

Japan war das zuletzt entstandene imperialistische Land, zur Macht aufgestiegen, als die Welt sich schon im Stadium des Imperialismus befand. Daraus resultierten allerdings Deformationen in seiner politischen und ökonomischen Struktur: Während es einerseits eine hochgradige Konzentration des Kapitals wie in den zaibatsu-Konzernen ${ }^{2}$ und eine weit 
fortgeschrittene Entwicklung der Produktivkräfte, repräsentiert etwa durch die den asiatischen Markt beherrschende Baumwollindustrie, aufwies, hielt es auf der anderen Seite politisch das Tennosystem aufrecht - mit dessen festgefügten, »absolutistischen« politischen Kompetenzen. Dazu kam eine unausgeglichene Gesellschaftsstruktur, in der die Kleinbauern mehr als die Hälfte der Erwerbsbevölkerung ausmachten und die Pachtbauern eine $\mathrm{Na}$ tural-Bodensteuer in der Höhe von mehr als 50\% des Ernteertrags zu entrichten hatten. Die Thesen von 1932 faßten dies in die folgenden Formeln:

1. Das Tennosystem nach den Meiji-Reformen ist eine absolutistische Monarchie.

2. Dieses Tennosystem stützt sich einerseits auf die Grundbesitzerschicht als eine parasitär-feudale Klasse, andererseits auf eine Bourgeosie, die gerade im Begriff steht, rasch zu Reichtum und Überfluß zu gelangen.

3. Dementsprechend ist das erste Ziel der japanischen Revolution die Zerschlagung des Staatsapparats des Tennosystems, d.h. eine bürgerliche Revolution, worauf dann die sozialistische Revolution folgt: die Revolution müsse also einen Prozeß von zwei Etappen oder Stufen durchlaufen.

Die hervorragenden Köpfe unter den marxistischen Ökonomen Japans konzentrierten sich nun darauf, durch empirische Analysen des japanischen Kapitalismus die wissenschaftliche Richtigkeit dieser Thesen nachzuweisen, um so die politische Strategie der KPJ rational zu begründen. Das Ergebnis dieser Anstrengungen war die insgesamt sieben Bände umfassende »Entwicklungsgeschichte des japanischen Kapitalismus« (1932/33). Die Verfasser dieses Werkes - Eitaro Noro (1900-1934), Moritaro Yamada (1897-1980), Goro Hani (1901 - 1983), Yoshitaro Hirano (1897-1980) und Shiso Hattori (1901-1956) - wurden danach die »Vorlesungen-Gruppe« (Koza-Gruppe) genannt, was auf den genauen Titel »Vorlesungen zur Entwicklungsgeschichte des japanischen Kapitalismus« zurückgeht. Diese Gruppe bildete seither die Hauptströmung unter den an der marxistischen Ökonomie orientierten Forschern. Deren Hauptthese lautete, daß es sich bei der Meiji-Reform insgesamt lediglich um eine Umwälzúng handelte, die das rein feudale Grundeigentum in semi-feudales Grundeigentum reorganisiert hätte, und daß die Besonderheit des japanischen Kapitalismus darin bestünde, daß er auf der Basis der Frontarbeit von halbleibeigenen Kleinstpächtern und halb-sklavenmäßiger Lohnarbeiter systematisch einen riesigen Militärapparat errichtet habe.

Aber es gab auch viele marxistische Wirtschaftswissenschaftler, für welche die von der Koza-Gruppe vertretene Hauptthese keine ausreichende Überzeugungskraft besaß. Mit ihrer Kritik an der Theorie der Koza-Gruppe vertraten sie die Ansicht, die Meiji-Reform sei eine bürgerliche Revolution gewesen, folglich sei die japanische Gesellschaft nach dieser Reform eine vollständig kapitalistische Gesellschaft, und das Ziel der politischen Umwälzung müsse in der sozialistischen Revolution bestehen. Wie hoch auch immer der von den Pachtbauern zu entrichtende Grundzins sei, es handele sich dabei um ein Resultat der heftigen Konkurrenz der Klein- und Kleinstbauern, und die feudalen Sozialbeziehungen mit dem Tennosystem an der Spitze seien lediglich feudale Relikte, die mit der Entwicklung des Kapitalismus Schritt für Schritt untergehen würden. Folglich sei die japanische Gesellschaft, wie feudalistisch sie auch immer aussehen mag, in ihrem Wesen eine kapitalistische Gesellschaft, und ihre sozialistische Umwälzung könne nur in einem Schritt erfolgen (»Ein-Stu- 
fen-Revolution $<$ ). Da diese Leute sich um eine Zeitschrift namens $»$ Rono $($ ( $)$ Arbeiter und Bauern «) gesammelt hatten, wurden sie in der Folge die »Arbeiter- und Bauern-Gruppe« (Rono-Gruppe) genannt. Zu ihr gehörten die Politiker Toshihiko Sakai (1870-1933), Hitoshi Yamakawa, Tsunao Inomata (1889-1942), Kanson Arahata (1887-1981), und Wissenschaftler wie Hyoe Ouchi (1888-1980), Tamizo Kushida (1885-1934), Itsuro Sakisaka (1897-1985). Die Theorie der Rono-Gruppe wurde von der Proletarischen Massenpartei (1928 gegründet) und der (Gesamtjapanischen) Arbeiter-Bauern-Massenpartei (gegründet 1931) aufgegriffen. Seit dieser Zeit wurde die marxistische Theorie in Japan in heftigen Kontroversen zwischen der Koza-Gruppe, die besonderes Gewicht auf den feudalen Charakter der japanischen Gesellschaft und die Rolle des Tennosystems legte, und der RonoGruppe, die den kapitalistischen Charakter der japanischen Gesellschaft betonte, entfaltet. Im Prozeß dieser Auseinandersetzungen machten auch empirische Untersuchungen über die Agrarökonomie um die Zeit der Meiji-Restauration 1867 und über den Entwicklungsstand der Industrie Fortschritte. Als Voraussetzungen für diese Analysen wurden Studien über die Wertlehre, die Lehre von den Reproduktionsschemata, die Lehre von der Grundrente u.a. vertieft.

Aber mit dem »Mandschurischen Zwischenfall $1931^{3}$ begann eine rasch fortschreitende Faschisierung der japanischen Gesellschaft. Studien der marxistischen Wirtschaftswissenschaft wurden nun gewaltsam unerdrückt. Im Jahre 1936 wurden, angefangen mit Moritaro Yamada, rund 30 Forscher der Koza-Gruppe verhaftet, 1937 etwa 400 Aktivisten der Rono-Gruppe, unter ihnen Itsuro Sakisaka. 1938 kam es dann zur Verhaftung von 41 marxistischen Ökonomen aus dem akademischen Bereich, die selber keine direkte Beziehung zur sozialistischen politischen Bewegung hatten. Selbst denjenigen, die in den Prozessen, die wegen des Verdachts des Verstoßes gegen das "Gesetz zur Aufrechterhaltung von Ruhe und Ordnung «geführt wurden, ohne Verurteilung davonkamen, war es unmöglich, wieder an die Universität zurückzukehren. Nach der Eröffnung des Pazifischen Krieges wurden fast alle marxistischen Wirtschaftswissenschafler aus den Universitäten vertrieben oder hinausgesäubert; auch außerhalb der Universitäten war die Zeit für marxistische Forschungen beendet. Alle möglichen Forschungen und Publikationen über marxisische Fragen wurden konsequent unterdrückt; den marxistischen $W$ issenschaftlern blieb nichts anderes übrig, als sich in private Institute zurückzuziehen und sich in Schweigen zu üben.

\section{Die Entfaltung der Uno-Theorie}

Der japanische Faschismus hat den Marxismus mit aller Gewalt unterdrückt. Doch Marxisten, die sich durch eine Erklärung der Staatsdoktrin des japanischen Faschismus unterwarfen und auf Forschungen auf Grundlage der Marxschen Theorie verzichteten, retteten damit ihr Leben. Trotzdem gab es unter ihnen welche, die im Gefängnis starben; aber die Mehrheit der marxistischen Politiker und Wissenschaftler konnte das Kriegsende überleben. Als mit dem Ende des Zweiten Weltkriegs die bis dahin bestehende politische Herrschaftsstruktur zusammenbrach, kehrten folglich viele marxistische Wissenschaftler wieder an die Universitäten zurück, und marxistische Forschungen blühterı sowohl innerhalb 
wie außerhalb der Universitäten auf. Dies war die Situation, in der Kozo Uno (1897-1977) mit seiner »Drei-Stufen-Theorie« besondere Aufmerksamkeit erweckte. Er legte damit eine Methodik zur Reinterpretation der Marxschen Ökonomik vor.

Der marxistische Wirtschaftswissenschaftler Uno, der vor dem Krieg der Rono-Gruppe angehörte, wurde wegen Verstoßes gegen das »Gesetz zur Aufrechterhaltung von Sicherheit und Ordnung « verhaftet und von der Universität vertrieben. Wahrend des Krieges aber konnte er seine Studien zur Marxschen Ökonomik fortsetzen, während er in einem privaten Institut beschäftigt war. Im Verlauf dieser Arbeit systematisierte er seine $\gg$ Drei-Stufen-Theorie«: die allgemeine, reine Lehre (Lehre von den Prinzipien der Ökonomie), die Lehre von der Stufenfolge kapitalistischer Entwicklung, sowie die Analyse der konkreten Situation. Mit dieser Methode des Herangehens an die Analyse der kapitalistischen Realität negierte er sowohl die Thesen der Koza- als auch der Rono-Gruppe. Denn gemeinsam ist beiden Richtungen ja, daß sie im »Kapitak einen direkt übertragbaren Maßstab erblickten, mit dem man an die Analyse der Wirtschaft Japans herangehen könne. So behauptete die Rono-Gruppe, der japanische Kapitalismus würde sich, obschon er auch weiterhin verschiedene hinterherhinkende soziale Praktiken aufweise, derzeit in Richtung eines vollständig entwickelten Kaptalismus bewegen, wie er im »Kapitak aufgezeigt worden sei. Aber auch die Koza-Gruppe nahm »Das Kapital« zum Maßstab, wenn sie zum Ergebnis kam, daß man von Japan nicht als einer kapitalistischen Gesellschaft sprechen könne, solange die feudalen Herrschaftsbeziehungen im japanischen Dorf und das Tennosystem fortbestünden.

Uno zufolge ist dagegen »Das Kapital《 ein theoretisches System des »reinen Kapitalismus«, das die Entwicklung des Kapitalismus in England Mitte des 19. Jahrhunderts als eine Art »Modell« benutzt. Doch verfolge der Kapitalismus als globales System einen historischen Entwicklungsprozeß in den drei Stufen des Merkantilismus, Liberalismus und Imperialismus. Es war Lenins Schrift »Der Imperialismus als höchstes Stadium des Kapitalismus«, in dem die Stuktur des »Kapitalismus« im Stadium des Imperialismus in einem einfachen Modell gezeigt wurde. Die aktuelle Situation des Kapitalismus der einzelnen Länder kann nicht durch die unvermittelte Übertragung von Kriterien oder Maßstäben aus dem »Kapital« analysiert werden, sondern allein dadurch, daß »Das Kapital« (reine Theorie) vermittelt mit der Leninschen Imperialismus-Schrift (Stufentheorie) angewendet wird. Uno sagt, daß sowohl der Koza-Gruppe als auch der Rono-Gruppe dieser methodologische Blickwinkel der Vermittlung durch die Stufentheorie gefehlt habe. Nach Uno haben also »Das Kapitel« als reine Theorie des Kapitalismus, Lenins Imperialismusstudie als Stufenlehre sowie die Analyse der aktuellen konkreten Situation jeweils eine unterschiedliche Abstraktionsebene und lassen sich nicht derselben theoretischen Dimension zuordnen. In dieser Hinsicht habe auch Lenin geirrt, der das Monopol aus der im »Kapital« entwickelten Akkumulationstheorie ableitete. Unos Position vereinfachend könnte man sagen: die Notwendigkeit der Krisen im Kapitalismus wird in der reinen Theorie nachgewiesen, die Notwendigkeit des Krieges in der Stufenlehre, und die Notwendigkeit der Revolution werde durch eine politische Bewegung »bewiesen«, die zur Voraussetzung die Erkenntnis der Struktur des jeweiligen kapitalistischen Landes mit Hilfe der Analyse der aktuellen Situation hat. 
Wenn man nun diese Drei-Stufen-Theorie auf die Analyse des japanischen Kapitalismus anwendet, kann man seinen besonderen Charakter, der sowohl bei der Koza- als auch bei der Rono-Gruppe im Unklaren blieb, einfach erklären. Obwohl auf der einen Seite die japanische Wirtschaft eine derartige Entwicklung der Baumwollindustrie erreichte, daß diese den asiatischen Markt zu beherrschen vermochte, und die zaibatsu-Konzerne Monopolorganisationen in großem Maßstab formten, wurden andererseits auf dem Lande noch Jahresabgaben eingezogen in einer Höhe, die $50 \%$ der Ernteerträge überstieg, und waren mehr als die Hälfte der erwerbstätigen Wirtschaftsbevölkerung Kleinbauern. Es herrschte also die Situation eines merkwürdigen Nebeneinanders von Entwicklung und Hinterherhinken. Die bis dahin vorherrschende marxistische Wirtschaftswissenschaft konnte diese Struktur, der die Balance fehlte, nicht angemessen erklären. Den drei Stufen Unos zufolge aber ist dieses Nebeneinander als das Charakteristikum der raschen kapitalistischen Entwicklung eines Nachzüglerlandes im Stadium des Imperialismus leicht erklärbar. Da in dem Nachzüglerland Japan die groBe Industrie des Fabriksystems mit ihrer hohen organischen Zusammensetzung des Kapitals auf einen Schlag aus Europa und den USA importiert wurde, ohne daß das Land den vorbereitenden Prozeß der Stufen der einfachen Kooperation und der Manufaktur durchlaufen hätte, war, auch wenn andererseits der Kapitalismus sich rasch entwickelte, die Nachfrage nach Arbeitskräften vergleichsweise gering. Während dementsprechend die Dörfer durch die Warenwirtschaft rasch aufgelöst und dort überschüssige Arbeitskräfte hervorgebracht wurden, konnten die Städte die Arbeitskräfte, insbesondere männliche volljährige Kräfte, kaum absorbieren; in den Dörfern, die zu einem Sammelplatz der latenten Überbevölkerung geworden waren, verschärften die Kleinbauern die Konkurrenz um Pachtland, wodurch die Bodenrenten in außerordentliche Höhen getrieben wurden. Auf dieser Grundlage wurde dann die feudale Grundbesitzerherrschaft perpetuiert. Da die Niedriglohnarbeiter und die armen Kleinbauern nur eine geringe effektive Nachfrage entfalten konnten, das Kapital aber mit hochentwickelten Produktivkräften produzierte, war Überproduktion die Folge. Die Inlandsmärkte waren also zu klein, so daß das Kapital auf der Suche nach überseeischen Märkten zum imperialistischen Vorrücken gezwungen war. Weil die herrschende Klasse im Inland die Arbeiterbewegung und die sozialistische Bewegung unterdrücken und die öffentliche Meinung für die Aggression gegen andere Länder vereinheitlichen mußte, war sie dazu gezwungen, die absolutistische Herrschaft des Tennosystems aufrechtzuerhalten. Hierin liegt der Grund dafür, daß in Japan die Entwicklung des Kapitalismus, anstatt zu Liberalismus und Demokratie zu führen, vielmehr umgekehrt die reaktionäre Staatsgewalt des Tennosystems noch verstärkt hat. Also ist das Tennosystem nicht einfach eine absolutistische Staatsgewalt, wie die Koza-Gruppe lehrte, noch ein feudales Relikt - was die Rono-Gruppe behauptete -, sondern vielmehr ein mit der imperialistischen Entwicklung des sich spät entwickelnden japanischen Kapitalismus kompatibler Gewaltapparat.

Kozo Uno hat aus dieser Sicht seiner Drei-Stufen-Theorie »Das Kapital als reine Theorie und die »Imperialismus«-Schrift als Stufenlehre theoretisch umgearbeitet; außerdem hat er die Begründung einer weder von politischer Praxis noch von Ideologie in Beschlag genommenen objektiven Sozialwissenschaft gefordert. Davon ausgehend hat er selbst in der Hochblüte des Stalinismus nach 1945 die Fehler der Stalinschen Ökonomik hervorgehoben und sich in der Kritik an den Analysen des japanischen Kapitalismus durch die KP-orientierten 
Wissenschaftler eine davon abweichende wissenschaftliche Haltung bewahrt. Deshalb näherten sich nach der »Entstalinisierung « 1956 viele Forscher und Studenten, die sich von der Kommunistischen Partei gelöst hatten — die hatte ihre Autorität als Avantgarde-Partei verloren -, der Theorie Unos. Das Resultat war, daß von den etwa 1000 Mitgliedern der Vereinigung der marxistischen Ökonomen in Japan (»Wissenschaftliche Vereinigung für Wirtschaftstheorie « - Keizai Riron Gakkai) ca. $20 \%$ der Uno-Richtung zugerechnet werden konnten.

\section{Der japanische Kapitalismus nach dem $\mathbb{Z}$ weiten Weltkrieg and die marxistische Wirtschaftswissenschaft}

Das Oberkommando der Alliierten Streitkräfte, die Japan Ende 1945 besetzt hatten, ging davon aus, daß das halbfeudale Grundeigentum, auf das sich die parasitären Grundbesitzer stützen konnten, die Monopolstellung der zaibatsu-Konzerne, und die staatlich-autoritäre Unterdrückung der Arbeiter Ursachen für die imperialistische Expansion des japanischen Kapitalismus waren. Die Besatzungsmacht veranlaßte daher die japanische Regierung, das politische System zu demokratisieren und zu diesem Zweck eine neue Verfassung zu akzeptieren. Vor allem wurden drei Maßnahmen zur Demokratisierung durchgesetzt: die Landreform, die Auflösung der zaibatsu sowie die Arbeitsreform. Davon wurde besonders die Landreform in konsequenter Weise durchgeführt: $81 \%$ des Pachtlandes wurden freigegeben; die Pachtbauern, deren Anteil an allen bäuerlichen Haushalten 26,6\% ausgemacht hatte, nahmen bis auf einen Anteil von 7,9\% ab, und mehr als die Hälfte aller Bauern wurden nunmehr als Landbesitzer selbstwirtschaftende Bauern. Da zudem während dieser Landreform die Inflation rapide fortschritt, kam der Zwangsverkauf von Grund und Boden zu amtlich festgesetzten Preisen für die Grundbesitzer beinahe einer entschädigungslosen Enteignung gleich.

Auf diese Weise wurde das parasitäre Grundbesitzertum, das von der Koza-Richtung als die »halb-feudale Basis« des japanischen Vorkriegskapitalismus betrachtet worden war, so gut wie völlig aufgelöst. Die Kommunistische Partei Japans jedoch, die 1951 den Anweisungen der Kominform gefolgt war und ihr $»$ Neues Programm« angenommen hatte, behauptete, Japan sei durch den amerikanischen Imperialismus zu einer Kolonie gemacht worden, und auch die Landreform würde das halb-feudale Grundeigentum lediglich reorganisieren, im Grunde aber fortbestehen lassen. Die mit der KPJ sympathisierenden marxistischen Theoretiker setzten alle Kraft daran, das Fortbestehen dieses Grundeigentums auf dem Lande nachzuweisen, auch indem sie nun die elf Bände umfassenden $»$ Vorlesungen zum japanischen Kapitalismus« herausgaben. Doch mangelte es dieser These wegen ihrer Entfernung zur tatsächlichen Wirklichkeit der Landreform offenkundig an Überzeugungskraft. Da außerdem die nach den Leitlinien der KPJ ausgerichtete Taktik überall Schiffbruch erlitt, war die Partei 1955 selber zu einem Kurswechsel gezwungen und mußte ihr „Neues Programm《 fallenlassen. Hinzu kam die erwähnte Entstalinisierung im Jahre 1956, die zu einem weiteren Autoritätsverlust der KPJ führte und den Auflösungsprozeß der Koza-Gruppe in der Wirtschaftswissenschaft entscheidend beschleunigte. 
In der Kommunistischen Partei verstärkten sich seit der 6. Nationalen Konferenz 1955 in beträchtlichem Maße Tendenzen des Parlamentarismus. Damit ging einher, daß die Partei sich immer mehr einer Richtung zuwandte, die von ihrer bislang verfolgten Strategie, welche auf der Theorie der Koza-Gruppe aufgebaut hatte, völlig verschieden war. Auch ein Teil der Theoretiker der Koza-Gruppe löste sich aus diesen Gründen von den ursprünglichen Thesen und verfolgte nun den Kurs der sogenannten 》strukturreform《. Diese Konzeption stützte sich auf die Theorie des Staatsmonopolistischen Kapitalismus des Deutschen Kurt Zieschang (DDR). ${ }^{4}$ In ihr wurde die Behauptung aufgestellt, die wachsende Bedeutung staatlicher Finanzpolitik, wie sie etwa in der New Deal-Politik der USA zum Ausdruck kam, die Verstärkung der Kontrolle des Staates über die Wirtschaft auf Basis eines kontrollierten Währungssystems, die Verstaatlichung von Schlüsselindustrien etc. würden hoch entwickelte Sozialisierungstendenzen anzeigen, die der fortgeschrittenen Entwicklung der Produktivkräfte entsprächen. Die Vertreter der »Strukturreform《-Theorie glaubten nun, daß unter dieser Voraussetzung der gestiegenen Bedeutung des staatlichen Zentrums in der Wirtschaft nach und nach eine sozialistische Transformation der Produktionsverhältnisse durch das Einwirken der demokratischen und sozialistischen Kräfte auf die Organe des Staates verwirklicht werden könnte.

Die Lehre von der »Strukturreform«mußte natürlich als politische Strategie zu einem Kurs der Reformen innerhalb des Rahmens des Parlamentarismus und zur Negierung einer gewaltsamen Revolution und der Diktatur des Proletariats führen. Die »Strukturreformer《, die sich von der KPJ gelöst hatten - die inzwischen eine Politik des Parlamentarismus verfolgte, aber gleichwohl noch an dem ihr eigenen nationalistischen Standpunkt festhielt (»nationalistisch« hier verstanden im Kontext einer politischen Strategie der »nationalen Befreiung « von der Vorherrschaft der USA - W.S.) - stimmten nun mit Saburo Eda (1907-1977) u.a. Verfechtern der »Strukturreform《 innerhalb der Sozialistischen Partei Japans (SPJ) überein. Man bemühte sich, die SPJ, deren leitende Prinzipien sich im Marxismus der Rono-Gruppe fanden, auf den Kurs der »Strukturreform « zu bringen, scheiterte damit aber letztlich. Die Linkskräfte in Japan lehnten Edas Vorstellung von einem neuen Sozialismus ab, den dieser in der Formulierung zusammengefaßt hatte: »Eine Verbindung von amerikanischem Lebensstandard, sowjetischen sozialen Garantien, britischer parlamentarischer Demokratie und japanischer Nachkriegs-, d.h. Friedensverfassung«. Dieses Auseinanderklaffen von Herrschaft des Parlamentarismus in der Realpolitik und Festhalten an der leninistischen Revolutionstheorie im Bereich der politischen Slogans bildete das Charakteristikum der Linkskräfte in Japan nach dem Krieg und galt sowohl für die KPJ als auch für die SPJ.

In Wirklichkeit hatte sich der größte Teil der Linkskräfte zwischen 1950 und 1960 unauf hörlich mit nicht allzu fruchtbaren Debatten befaßt, wie etwa der, ob es sich in Japan nun um koloniale Abhängigkeit oder imperialistische Selbständigkeit handele, ob es um eine Volkspartei oder eine Klassenpartei ginge, ob friedliche Reform oder gewaltsame Revolution der richtige Weg sei. Bei jeder Wahl aber sicherte sich die Konservative Partei die Mehrheit der Stimmen, während von den sozialistischen Kräften die Mauer von einem Drittel der Wähler nicht übersprungen werden konnte. Mit Ausnahme einer nur wenige Monate andauernden Koalitionsregierung unter der Sozialistischen Partei (1947/48) hat 
die Konservative Partei in der japanischen Nachkriegspolitik durchgängig die Regierung gestellt - ein für die »freie Welt« seltenes, einzigartiges Phänomen. Einer der Hauptgründe hierfür liegt darin, daß die linken Kräfte und die marxistischen Theoretiker in Japan den neuen historischen Charakter der Entwicklung des japanischen Nachkriegskapitalismus nicht klar erfassen und sich außerdem dem Banne der These von der »allgemeinen Krise « des Kapitalismus, wie sie in der Komintern vertreten wurde, nicht entziehen konnten. Tatsächlich hatte Uno bei seinem Tode so gut wie keine systematischen Analysen des gegenwärtigen Kapitalismus nach dem Ersten Weltkrieg hinterlassen, und Tsutomu Ouchi (geb. 1918), von dern es hieß, er habe an Stelle Unos die Lehre vom Gegenwartskapitalismus in der Uno-Theorie entwickelt, hatte in seiner eigenen Lehre vom staatsmonopolistischen Kapitalismus prognostiziert, daß der heutige Kapitalismus im Grunde durch Niedrigwachstum und Stagnation charakterisiert sei, folglich auch das hohe Wirtschaftswachstum nach dem Zweiten Weitkrieg lediglich ein zeitweiliges Phänomen und demgemäß bald mit einem Absinken in langfristige Stagnation zu rechnen sei. ${ }^{5}$ Aber etwa von 1961 an, als diese Voraussage gemacht wurde, ging der japanische Kapitalismus in seine zweite Boomphase über, und diese hielt dann bis zur Ölkrise 1973 an. Die Voraussage Ouchis hat sich als völliger Mißgriff erwiesen.

So scheiterten die sozialistischen Parteien in ihrem Bemühen, ihre Kräfte auszuweiten, und korrespondierend dazu versagte auch die marxistische Wirtschaftswissenschaft in der Analyse der Mechanismen des hohen Wirtschaftswachstums des japanischen Nachkriegskapitalismus. Sich gegenseitig beeinflussend waren beide in entscheidender Weise daran beteiligt, daß der Marxismus seiner Autorität verlustig ging.

\section{Hohes Wirtschaftswachstum und gegenwärtiger Kapitalismus}

Nach dem Zweiten Weltkrieg hat sich die japanische Wirtschaft in ungefähr zehn Jahren von ihrem völligen Zusammenbruch wieder erholt. Im großen und ganzen zeigten um das Jahr 1955 die verschiedenen Wirtschaftsindikatoren an, daß Japan im wesentlichen den Standard der Vorkriegsperiode (von 1935) wieder erreicht hatte. Und von ca. 1955 bis ca. 1973 (dem Zeitpunkt der ersten Ölkrise) konnte es ein hohes Wirtschaftswachstum mit jährlichen Steigerungsraten des Bruttosozialprodukts (GNP) von durchschnittlich $10 \%$ verzeichnen. Da das Wachstum in der Vorkriegsperiode im Durchschnitt bei 3 bis $4 \%$ lag, war dieses hohe Wirtschaftswachstum nach dem Krieg für Japan historisch eine erstmalige Erfahrung. Auch im internationalen Vergleich gibt es in der Geschichte des kapitalistischen Weltsystems kein Beispiel ähnlicher Dynamik. Hierdurch jedenfalls hat die japanische Wirtschaft ihren Charakter völlig verändert.

Vergleichen wir einmal die Indexzahlen von 1955 und 1970: 1955 betrug das BSP 24 Mrd. US-Dollar (d.h. 1/15 desjenigen der USA, die Hälfte des westdeutschen); das Bruttoinlandsprodukt pro Kopf betrug 204 US-Dollar - damit lag es an 35. Stelle in der Gruppe der westlichen Länder. Weil die Agrarbevölkerung innerhalb der gesamten erwerbstätigen Bevölkerung 37,9\% ausmachte, kann man Japan zu jenem Zeitpunkt getrost als »Schwellenland « bezeichnen. 
1970 jedoch lag das BSP bei $206 \mathrm{Mrd}$. Dollar und das BIP pro Kopf bei 2000 Dollar. Letzteres ist damit nur halb so groß wie dasjenige in den USA; Japan hat aber Italien hinter sich gelassen (1900 Dollar) und ist England auf den Fersen (2200 Dollar). Die Agrarbevölkerung hat dramatisch abgenommen: sie macht nur noch $16,1 \%$ aus, d.h. weniger als die Hälfte des Anteils von 1955. Diese Zahl bewegt sich in der Größenordnung von Frankreich und Italien, aber die Abnahmerate der im Agrarbereich Erwerbstätigen ist die höchste in den fortgeschrittenen Ländern.

Den Kern dieser rasch wachsenden Wirtschaft bildeten die mit der Produktion langlebiger Konsumgüter befaßten Industriezweige: Automobilindustrie, elektrische Haushaltsgeräte u.a. Die Anzahl der von der japanischen Automobilindustrie hergestellten Fahrzeuge belief sich 1955 auf magere 73.400 Stück, stieg aber bis auf 5,884 Mio. 1971 an, d.h. um das 80-fache. Bei den Anlageinvestitionen ergibt sich ein ähnliches Bild: sie lagen 1955 bei $4 \mathrm{Mrd}$. Yen, 1971 jedoch waren sie auf 249,8 Mrd. Yen angestiegen - um das 62 fache.

Bei der Arbeitskraft wandelte sich die Situation von einer des chronischen Überflusses zu einer der Vollbeschäftigung; die Rate der Arbeitslosen ging auf 1 bis $2 \%$ zurück. Unter diesen Bedingungen von Vollbeschäftigung stiegen die Löhne jährlich um rund 10\%; innerhalb von 20 Jahren verdreifachten sie sich beinahe. So kam es, daß sich annähernd $90 \%$ der Bevölkerung in Umfragen den Mittelschichten zuordneten.

Während sich die konservativen Kräfte in der Liberal-Demokratischen Partei (LDP) zusammenschlossen, war die Opposition in Sozialistische Partei, Kommunistische Partei, Demokratisch-Sozialistische Partei, sowie die neobuddhistische »Partei für eine saubere Regierung « (Komeito) gespalten. In den Wahlen eroberte die LDP durchweg die absolute Mehrheit und stellte damit fortlaufend die Regierung. Dieses politische System Nachkriegs-Japans wird gewöhnlich als das »1955-er System « bezeichnet (in diesem Jahr vereinigten sich die verschiedenen konservativen Parteien wie auch der linke und der rechte Flügel der Sozialisten - W. S.), doch bildete seine eigentliche Grundlage eine durch hohe Wachstumsraten gekennzeichnete Wirtschaft. In diesem »1955-er System《 wurden zunächst einmal die durch die Landreform befreiten Bauern vollständig in den bestehenden Rahmen integriert, indem auf dem Lande beträchtliche Geldsummen als Subventionen ausgestreut und die Getreidepreise von der Regierung abgestützt wurden (im wesentlichen betrifft dies den Reis). Es wurden dann aber auch die Industriearbeiter durch Vollbeschäftigung und kontinuierliche Lohnerhöhungen ins System einbezogen. Ihre sich auf Umwälzung richtenden Energien wurden von der einmal im Jahr stattfindenden »Frühjahrsoffensive« für Lohnerhöhungen (shunto) absorbiert, und während in der Gewerkschaftsbewegung der Sozialismus weiterhin als Slogan hochgehalten wurde, bestand die wahre Gesinnung dieser Arbeiter in der Unterstützung des »1955-er Systems«. Studenten und junge Arbeiter, die mit den wachsenden konservativen Tendenzen unter den Bauern und Arbeitern sowie mit dem Umkippen der sozialistischen Parteien in eine systemimmanente Opposition unzufrieden waren, radikalisierten sich in der zweiten Hälfte der 60er Jahre - wobei der Japanisch-Amerikanische Sicherheitsvertrag den Anlaß des Problems lieferte. Sie entfachten die ganz Japan erfassenden Universitätskämpfe und schufen in den Städten sogar eine Situation des Aufruhrs. Aber auch diese Bewegungen verloren durch Flugzeugentführungen und Bombenterror, durch interne Spaltungen und gewaltsame Auseinandersetzungen zwischen den Gruppierungen die Unterstützung der Öffentlichkeit und 
wurden durch den Einsatz der Polizeigewalt sowie durch Verabschiedung eines Gesetzes zur Kontrolle der Universitäten schließlich zum Schweigen gebracht.

1971 brach durch die Beendigung der Goldbindung des Dollar das IWF-System von Bretton-Woods faktisch zusammen, und 1973 kam es zur Ölkrise. Damit waren die goldenen Zeiten des hohen Wirtschaftswachstums global beendet. Auch der japanische Kapitalismus trat in eine Periode langanhaltender Rezession ein, aber Arbeiter- und sozialistische Bewegung waren auch weiterhin von Kraftlosigkeit gekennzeichnet. Die japanische Betriebsform mit ihren Merkmalen der lebenslänglichen Anstellung, der Entlohnung nach Dienstalter und der unternehmensinternen Gewerkschaften — die Grundlage für das Zugehörigkeitsbewußtsein der Arbeiter gegenüber dem Unternehmen - wurden auch in der langen Phase des hohen Wachstums aufrechterhalten; jetzt, mit dem Eintreten in die Rezessionsphase, haben diese Institutionen bei den Arbeitern sogar umgekehrt die »hingebungsvolle Haltung « dem Unternehmen gegenüber verstärkt; die Arbeiter versuchen, auf diese Weise die eigene Beschäftigung zu verteidigen.

Aber für den Stillstand der sozialistischen Kräfte im heutigen Japan ist auch das Stagnieren der marxistischen Wirtschaftswissenschaft mitverantwortlich. Denn fast alle marxistischen Theoretiker waren einerseits unfähig, das beschriebene hohe Wirtschaftswachstum zu prognostizieren und haben fortwährend das baldige Kommen einer Weltwirtschaftskrise und langanhaltender Stagnation angekündigt; andererseits haben sie nicht vermocht, die von diesem hohen Wirtschaftswachstum hervorgebrachte Umweltzerstörung und Landflucht sowie Übervölkerung in den Ballungsgebieten, den Zusammenbruch des IWF-Systems, die Ölkrise und die Rohstoffrage, das Nord-Süd-Problem u.a.m. als strukturelle Mängel des gegenwärtigen Kapitalismus herauszuarbeiten und theoretisch einzuordnen. Tatsächlich hat sich gezeigt, daß Japan als Preis für sein Wirtschaftswachstum die Produktion zahlreicher inländischer Rohstoffe, einschließlich der Agrarproduktion und von Steinkohle, eingestellt hat, so daß es im Bereich Rohstoffe und Energie fast vollständig vom Ausland abhängig wurde. (Z.B. betrug 1975 die Bereitstellung von Energie aus Inlandsproduktion nur $12 \%$, während die Einfuhren sich auf $88 \%$ beliefen.)

Als Ergebnis der Konzentration von 80\% der Schwer- und Chemieindustrie in Kombinaten an der Küste zum Pazifik konzentrieren sich 80\% der Bevölkerung in den dort liegenden Städten. Während die Dörfer weiterhin unter der Landflucht leiden, explodieren die Bodenpreise in den Städten (jährliche Steigerungsrate: 30\%). Besonders an der Pazifikküste werden die Umweltschäden immer größer, die Verschmutzung der Natur wird immer unerträglicher, so daß Japan ein Vorbild-Archipel für Umweltzerstörung geworden ist. Zudem hat die Anzahl der in Japan produzierten Kraftfahrzeuge diejenige in den USA hinter sich gelassen, womit zugleich auch die Motorisierung in Japan selbst ihre bisherigen Grenzen überschreitet. Die Zahl der jährlichen Verkehrstoten und -verletzten beläuft sich inzwischen auf 700000 bis 800000 Menschen.

Ferner vollzog sich hinter diesem Prozeß des hohen Wirtschaftswachstums die fortschreitende Verelendung der Entwicklungsländer der südlichen Hemisphäre: von ihnen wurden gewaltige Rohstoffmengen zu niedrigen Preisen aufgekauft, aber zugleich bildeten sie dann die Absatzmärkte für die teuer verkauften Produkte der Schwer- und Chemieindustrie. Die 60er Jahre der Prosperität des Nordens sind die 60er Jahre des Scheiterns des Südens. Dies 
wäre im Unterschied zu dem gewaltsamen kolonialen Ausplünderungsmechanismus durch den Imperialismus als friedlicher Ausplünderungsmechanismus des gegenwärtigen Kapitalismus zu bezeichnen. Das mit den angesprochenen Tendenzen zur Motorisierung zusammenhängende Wirtschaftswachstum hat im übrigen auch die sozialen Lebensbedingungen und das Familienleben der Beschäftigten stark beeinflußt. In den Haushalten überwiegt immer mehr die Kernfamilie, und mit der heute mehrheitlich anzutreffenden Doppelbeschäftigung von Mann und Frau haben sich auch die Haushalte weiter aufgelöst: es ist eine Zunahme jugendlicher Delinquenz zu beobachten, wie andererseits immer mehr Alte allein wohnen. Gewalt in den Familien wie in den Schulen ist inzwischen weit verbieitet. Kurzum, mit dem Steigen der Einkommensniveaus ist auch der letzte gemeinschaftliche Zusammenschluß in der Gesellschaît weitgehend zerstört worden. Wahrscheinlich kann man die epidemische Verbreitung derartiger gesellschaftlicher Krankheitsphänomene auf folgende spezifische Struktur zurückführen: Der Kapitalismus der Gegenwart verschwendet in rascher Folge seine überschüssigen Produktivkräfte und schafft, indem er die Nachfrage der Verbraucher stimuliert, fortwährend neue Bedürfnisse; er ist gezwungen, die Öberproduktion an Waren in die Märkte hineinzudrücken. Dieser Gegenwartskapitalismus beutet also nicht nur einfach die Arbeiter im Wirtschaftsprozeß aus, sondern nützt Leib und Seele von ihnen und alle Bereiche ihres Lebens als Quelle der Verwertung des Kapitals, indem er die Bedürfnisse der Arbeiter selber manipuliert. Und das Territorium, auf das sich seine Ausplünderung erstreckt, hat er, vermittelt über Welthandel und multinationale Unternehmen, in globalem Umfang ausgeweitet; die konsequent betriebene Ausplünderung schreitet in der friedlichen Form der (Entwicklungs-) Hilfe und des Exports der »Motorisierungs-Zivilisation « voran und ist weniger imperialistische Ausplünderung im früheren Sinn.

Um das Geheimnis dieser Ausplünderung des in allen Listen bewanderten Gegenwartskapitalismus zu entschlüsseln, sind $»$ Das Kapitak und die Leninsche Imperialismus-Schrift natürlich nicht (mehr) von Nutzen, aber auch nicht »Theorien der allgemeinen Krise« oder Theorien des »staatsmonopolistischen Kapitalismus«. Notwendig ist wohl ein völlig neues theoretisches System für diesen völlig neuen Typus der Kapitalakkumulation. Bedauerlicherweise haben die marxistischen Theoretiker bei diesem Unternehmen noch keinen Erfolg aufzuweisen.

Zur Zeit werden in Japan, um dieses theoretische Vakuum zu füllen, Versuche angestelit, interdisziplinäre Kulturanthropologie, Wirtschaftsanthropologie, Strukturalismus, Ökologie, Systemkomparatistik u.a. mit der marxistischen Wirtschaftswissenschaft zu verbinden. Aber all diese Versuche sind noch nicht bis zu einer systematischen Erklärung der Grundprobleme der heutigen Welt in ihrer Gesamtheit und ihrer historischen Bedeutung gediehen. Es scheint, daß die marxistische Wirtschaftswissenschaft in Japan parallele Erscheinungen mit der Stagnation der japanischen Wirtschaft nach der Periode des hohen Wirtschaftswachstums und der Aushöhiung ihrer Industrie ganz aufweist und noch nicht in der Lage ist, ihre Verwirrung zu überwinden. 


\section{Anmerkungen des Ubersetzers/der Redaktion}

1 Der japanische Begriff »Meiji ishin« bedeutet wörtlich: die mit Beginn der »aufgeklärten Regierung« einsetzende Erneuerung. Er bezieht sich im allgemeinen auf die ersten beiden Jahrzehnte der Ära Meiji (oder: Meiji-Periode) (1868-1912). Die jeweilige Ära beginnt mit der Thronbesteigung eines neuen Tenno, dem posthum der Name der $\ddot{A}$ ra gegeben wird. Daher spricht man ab 1912 von Kaiser Mutsuhito als dem »Meiji-Tenno«. - Im engeren Sinne wird mit »Meiji ishin « die Restauration des Kaisertums bezeichnet, also die Wiedereinsetzung des Tenno in seine Rechte nach Jahrhunderten faktischen Macht- und Autoritätsverlustes. Im weiteren Sinne sind über diese »Meiji-Reform《 als einer politischen Umwälzung hinaus die verschiedenen Reformen gemeint, die unter der Regentschaft des jungen Meiji-Kaisers von der neuen Regierung durchgeführt wurden und maßgeblich Japans Entwicklung zu einer industriekapaitalistischen Großmacht ermöglichten. Diese Reformen lösten eine soziale Umwälzung aus, wie sie andererseits durch endogene Entwicklungen der japanischen Gesellschaft und den äußeren Druck seitens der westlichen Großmächte mit Begitin des 19. Jahrhunderts angebahnt wurden. Neben den im Text erwähnten Maßnahmen ist die Verlegung der Hauptstadt von K yoto nach Edo und dessen Umbenennung in Tokyo (= östliche Hauptstadt) zu erwähnen; 1871 die Auflösung der Daimate, der rund 260 Lehnsfürstentümer unter den Daimyo, und ihre Umwandlung in Präfekturen des neuen Zentralstaats; die Abschaffung der Ständeordnung (1870-1876), die zum Verlust der Privilegien der Kriegerklasse (samurai) führte; 1873 die Reform der Grundsteuer. Die verschiedenen politischen und sozio-ökonomischen Reformmaßnahmen wurden unter zweierlei Zielsetzung durchgeführt: »Zivilisation und Aufklärung« und »ein reiches Land und eine starke Armee«.

2 zaibatsu (wörtlich: Finanzclique) bezeichnet ein großes Monopolkapital, das durch Verflechtungen einer Familie beherrscht wird, deren Wirtschaftsimperium sich über verschiedene Zweige im Industrie- und Bankensektor erstreckt. Beispiele vor 1945 waren die Konzerne der Mitsui, Mitsubishi, Sumitomo, Furukawa und Shibusawa. Die zaibatsu verfügten über beste Verbindungen zur staatlichen Bürokratie und seit dem »mandschurischen Zwischenfalk 1931 auch zum Militär. Im Oktober 1945 erging seitens der Besatzungsmacht der Befehl zu ihrer Auflösung, 1947 wurde das Antimonopolgesetz verabschiedet. Doch mit dem im Zuge des Kalten Krieges von den USA eingeschlagenen »reverse course« wurden die Ansätze zur Auflösung wieder rückgängig gemacht. Heute besitzen die zaibatsu wieder eine enorme Machtstellung in der japanischen Wirtschaft und Politik, wenngleich die alten Familien nicht mehr die Lenkung der Konzerne in ihren Händen haben.

3 Am 18.9.1931 provozierten Truppenteile der japanischen, in der Mandschurei als Schutztruppe stationierten Kwantung-Armee einen $» Z$ wischenfalk in der Nähe der Stadt Mukủen und eröffneten anschließend das Feuer auf chinesische Truppen. Damit war der Anlaß geschaffen, um mit Hilfe aus Korea herbeigeholter Verstärkungen in den nächsten drei Monaten sämtliche mandschurischen Provinzen zu besetzen. Im März 1932 wurde von Japan dann der Marionettenstaat »Mandschukuo« errichtet. Die japanische Regierung, die das eigenmächtige Vorgehen der Truppe zunächst mißbilligte, hatte bereits früher argumentiert, die ökonomische und politische Kontrolle über die Mandschurei sei notwendig, um einen Angriff Rußlands abwehren und die Sicherheit Chinas garantieren zu können. Im Grunde ging es aber darum, den chinesischen Widerstand gegen die japanische Truppenpräsenz endgültig zu brechen.

4 Kurt Zieschangs Theorie des staatsmonopolistischen Kapitalismus übte in den 60er Jahren einen gewissen Einfluß in Japan aus. Zieschang bestimmt den »staatsmonopolistischen Kapitalismus« als »eine Entwicklungsstufe der kapitalistischen Produktionsverhältnisse « und ordnet ihn einer höheren Ebene der kapitalistischen Entwicklung als der des Imperialismus zu. Er wird damit deutlich vom »klassischen« Imperialismus unterschieden. Vgl. K. Zieschang: Zu eirigen theoretischen Problemen des staatsmonopolistischen Kapitalismus in Westdeutschland, Berlin (DDR), 1957; A. Heinze u.a.: Aktuelle Probleme des staatsmonopolistischen Kapitalismus, Berlin (DDR), 1961. Zur Kritik siehe M. Wirth, Kapitalismustheorie in der DDR, Frankfurt/M., 1972. 
5 Die »Varga-Debatte« entzündete sich 1947 in der Sowjetunion an der Veröffentlichung von Eugen Vargas »Veränderungen in der kapitalistischen Wirtschaft in Gefolge des Zweiten Weltkriegs«. Varga meinte, daß sich der Kapitalismus zu stabilisieren vermöge; denn »keine Krise dauert ewig«. Er analysiert auch die Möglichkeiten der Bourgeoisie, »sozialistische Maßnahmen« durchzuführen, um ihre Herrschaft zu verlängern. Gegen Varga behauptete die Orthodoxie auf. der Grundlage der Stalin'schen Thesen, daß »das Gebäude des modernen imperialistischen Kapitalismus« nicht nur nicht »morsch « geworden sei, sondern unmittelbar vor dem Zusammenbruch stehe. Die Debatte um Vargas Thesen hatte großen Einfluß in allen kommunistischen Parteien. Vgl. Sowjetwissenschaft, Heft 1, 1947; Werner Petrowsky, Zur Entwicklung der Theorie des staatsmonopolistischen Kapitalismus, in: Probleme des Klassenkampfs, Heft 1, 1971 